\title{
Web Based Female Body Type Identification and Its Application
}

\author{
Su-yu Zhang \\ Division of Information Technology, Wenzhou Polytechnic, Wenzhou, China \\ Email: 23209695@qq.com
}

\begin{abstract}
Establishing the identification and application system of female body type on the basis of Web is to produce the clothing patterns conforming to the body size of human and meet the different demands of customers. A human body database was set up by collecting the data of young female in Zhejiang Province, and then the basic pattern database was set up in accordance with the basic pattern produced by garment enterprises. Finally the personalized garment pattern came into being by identifying the individual data through SVM. The system is carried out through the self-made software of network platform constructed by J2EE, and is effective in the application of enterprises.
\end{abstract}

Keywords: garment pattern, database, body type identification, SVM, web

\section{Introduction}

With the continuous development of modern society and economy, more and more customers not only require personalization in styles, but also hope to meet their own body type characteristics when buying clothes [1]. For consumers, clothing with the wrong size and body shape, no matter how satisfied they are, they can only give up; for manufacturers, the fit and matching of the clothing sold is not good, which will lead to a large backlog of clothing. It can be seen that the fit of clothing occupies a very important position in clothing design, and accurate body shape recognition and classification are the basis of clothing model and modeling design [2].

In order to meet the personalized design requirements of clothing models, this paper conducts detailed analysis on the body shape characteristic parameters of young female in Jiangsu and Zhejiang, using SVM (support vector machine) algorithm for recognition, and constructs a set of Web-based 3D female body shape recognition and personalized model generation The system can complete functions such as body shape recognition, 3D human body modeling and 2D pattern automatic generation. This system uses a J2EE architecture network platform for data transmission to meet the individual needs of consumers.

\section{Data Collection and Classification}

\subsection{Three-dimensional Human Body Data Collection and Input}

The author has analyzed in the reference [3] the needs of different regions in the national standards for body shape differences, consumers' demand for clothing fit, and clothing companies' demand for mass customization. This paper only conducts demand analysis for young female aged 19 to 35 in Jiangsu and Zhejiang. There are two types of data patterns entered: one is the basic personal information manually entered, which mainly includes the social background information and weight of the tested person; the other is the feature size information of the human body automatically generated by the three-dimensional measuring instrument [4].

According to the theory of mathematical statistics, when the sampling is simple random sampling and the sample size is large, the random variable approximately obeys the normal distribution. For random variables that do not follow a normal distribution, if $\mathrm{N}$ is quite large, the random variable will be very different from the standard normal distribution random variable. It is generally believed that $\mathrm{N}>50$. Therefore, the minimum sampling amount $\mathrm{N}$ value should be determined under certain conditions, and the determination of the $\mathrm{N}$ value depends on the selected statistics. The confidence level set for this measurement is $99 \%$ to maximize coverage of various body types. The minimum sample size $\mathrm{N}$ value can be calculated according to the following approximate formula: 


$$
N=\left(\mu_{\alpha} \times \frac{S}{\Delta}\right)^{2}
$$

\subsection{Female Body Type Classification}

\subsubsection{Female feature parameter extraction}

Taking female's waist line as the boundary, they are divided into upper and lower bodies, mainly using the following two methods to subdivide: One is to subdivide female's body shape from the overall shape. Taking the national size as the standard, based on the body type recognition of the chest and waist difference, this also enables the output of the body type classification results of the individual when using the national standard in the body type discrimination, which increases the practicality of the database. The second is to subdivide female's body shape from the local feature parts. The geometric features of important parts of female's body shape and the parts related to the design of clothing structure are mainly used to carry out research on the characteristic parameters of female's chest, back, buttocks and abdomen. See Table 1 for the comparison between the body shape of young female in Jiangsu and Zhejiang and the national sample of female.

Table 1. The comparison of the somatotype of young female in Jiangsu and Zhejiang with the national sample of female

\begin{tabular}{lcccc}
\hline \multicolumn{1}{c}{ Name } & Average height $(\mathrm{cm})$ & Average bust $(\mathrm{cm})$ & Average waist $(\mathrm{cm})$ & Average hip $(\mathrm{cm})$ \\
\hline $\begin{array}{l}\text { National Females' Body Shape } \\
\text { Sample }\end{array}$ & 156.50 & 84.06 & 69.74 & 90.37 \\
\hline $\begin{array}{l}\text { Body shape samples of young } \\
\text { Female in Jiangsu and Zhejiang }\end{array}$ & 160.16 & 84.67 & 67.54 & 89.18 \\
\hline Regional standard deviation & 5.12 & 4.37 & 4.76 & 4.16 \\
\hline
\end{tabular}

\subsubsection{SVM algorithm classification and recognition}

Based on the SVM is a system model belonging to the black box, so it only cares about the input and output of the object, not the specific structure of the object. The mapping relationship between input and output is realized through SVM. The specific system model identification steps are as follows: first use the input variable $\mathrm{x}$ and output variable y to train the SVM; then use the trained model to estimate the output variable; finally in the nonlinear system model, the input variable and the output variable are Non-linear functional relationship between SVM

$$
f(x)=\sum\left(\alpha_{i}-\alpha_{j}^{*}\right) K\left(x_{i}, x_{j}\right)+b
$$

SVM is used to model females' body size, and analysis and design are carried out through the relevant functions of the Matlab toolbox.

(1) Input and output parameters. Take the chest feature as an example, using the extracted six feature variables (under bust slope angle, front neck to breast angle, side neck to breast/ breast to waist, bust to bust / bust front, bust width/bust thickness, bust full), qualitatively analyze the three-dimensional shape of females' chest. Six feature parameters are used as the input of the training network, and the chest shape (full, medium, flat) judged by experts is used as the output of the training network. Since the training network cannot accept language information, the full, medium, and flat language variables as output need to be converted into discrete numerical variables. Set full to 3 , medium to 2 and flat to 1 .

(2) Data standardization. The kernel function of this system adopts the kernel of radial basis function:

$$
K\left(x_{i}, x_{j}\right)=e^{-\frac{\left\|x_{i}-x_{j}\right\|^{2}}{2 \sigma^{2}}}
$$

After normalizing the input and output data according to the maximum and minimum values of the input and output data, the standardized data is compressed between $[-1,1]$ in this experiment. 
Feature parameter selection of females' body shape is mainly carried out on four local parts of women: bust, flank, hip, and abdomen. The bust grade is $\mathrm{R}$, the flank grade is $\mathrm{C}$, the hip grade is $\mathrm{T}$, and the abdomen grade is $\mathrm{F}$. The number of body types is $\mathrm{S}=\mathrm{R} \times \mathrm{C} \times \mathrm{T} \times \mathrm{F}$. The index values of each part are: $\mathrm{R}=3, \mathrm{C}=2, \mathrm{~T}=3, \mathrm{~F}=2$. Females' body types are divided into three levels: flat, medium, and full according to the bust, which are represented by R1, R2, and R3 respectively; according to the flank, they are divided into two levels: medium and curved, which are represented by $\mathrm{C} 1$ and $\mathrm{C} 2$ respectively; according to the hips, they are divided into three levels: flat, medium, and full, which are represented by T1, T2, and T3; according to the abdomen, they are divided into two levels: medium and convex, which are represented by F1 and F2 respectively. In this way, according to the different values of the four parts, the females' body types are divided into $3 \times 2 \times 3 \times 2=36$ types. Figure 1 shows the combination of female body shape characteristics.

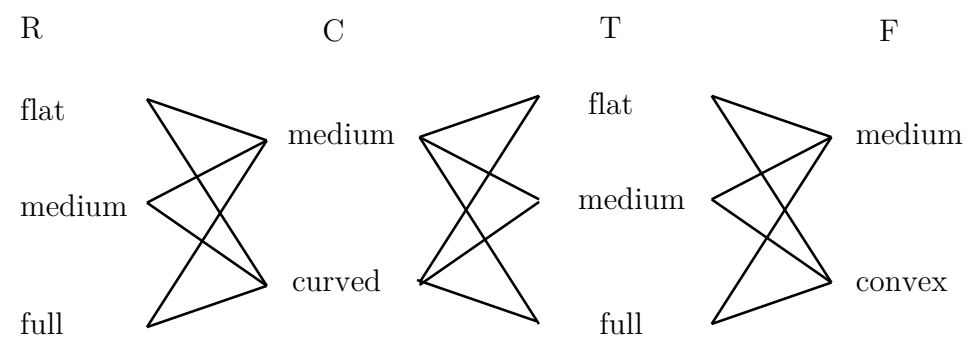

Figure 1. Females' body shape combination

\subsection{Personalized Template Generation}

It mainly completes the two functions of generating basic model data and personalized model data.

\subsubsection{Basic template generation}

Store the size of the basic model of the garment manufacturing company in the database, and then find out each key feature point according to the model graph, connect the points with a straight line and a Bezier spline curve to form a basic top and bottom model graph.

\subsubsection{Personalized template generation}

Using 160/84S15 as the standard female body size template, on the one hand, the size difference between the individual body size and the 160/84S15 standard body template size is formed; on the other hand, considering the effect of height, the national standard size difference is used. The sum of the two is the grade difference required for grading the basic model. Utilize the upper and lower M templates of the enterprise in the system template database, and scale the code according to the grade difference value to form a personalized template.

\section{System Programming and Implementation}

The web-based 3D female body shape recognition and personalized model generation system adopts J2EE architecture. The specific architecture is: WebWork+Spring+Hibernate, following the Model-ViewController (MVC) model, using the current popular network Programming technology-JSP as the main realization means (cooperating with JavaServlet, JavaBean, JavaScript, HTML, etc.), the integrated system function is realized under the eclipse development tool. The server-side operating system of the system is configured as Windows 10, and the database adopts Microsoft SQL Server 2019. The main interface of the online system for female body shape recognition and model generation is shown in Figure 2 . 


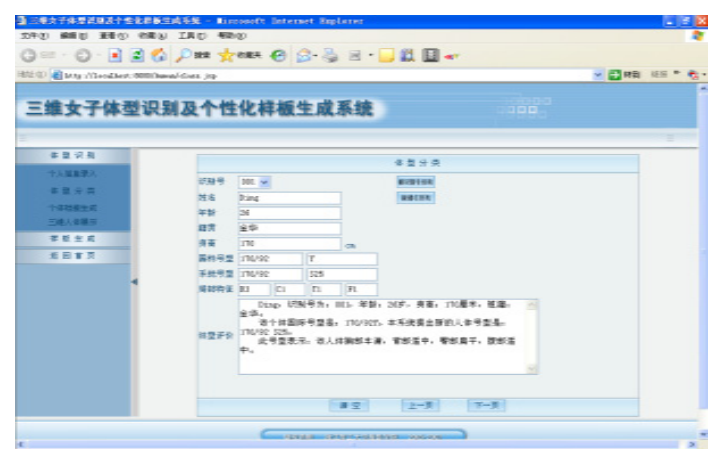

(a)

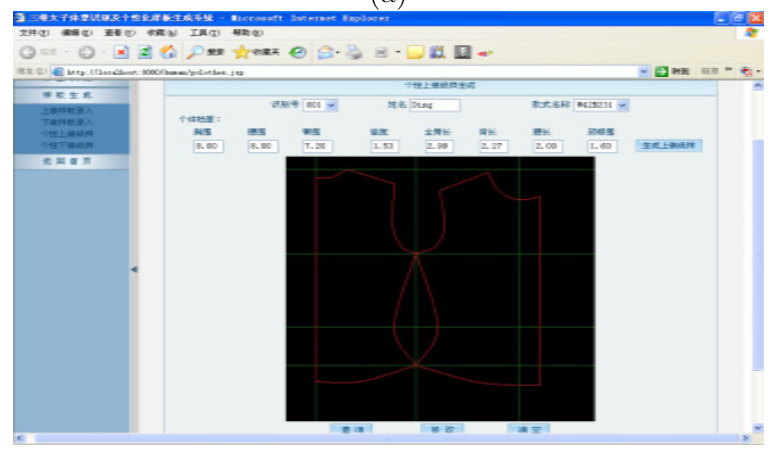

(c)

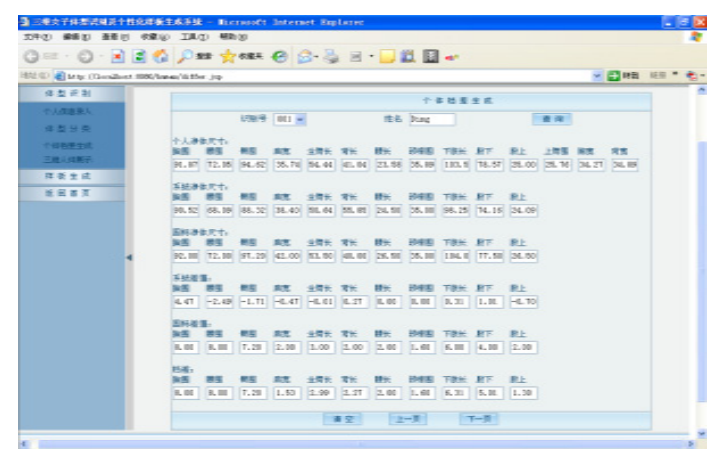

(b)

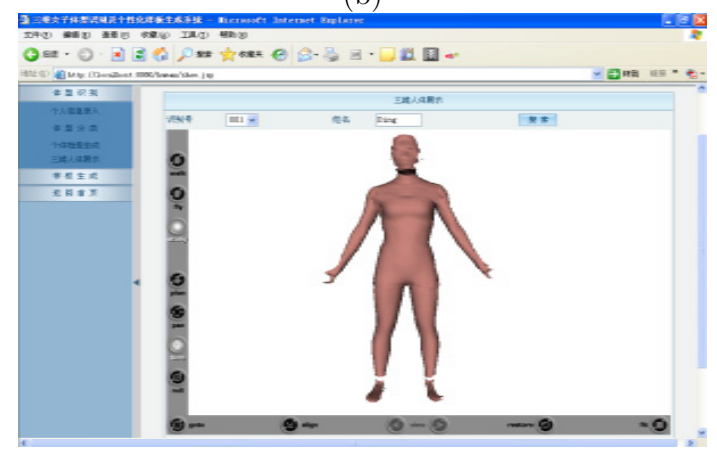

(d)

Figure 2. The main interface of the online system for female body shape recognition and model generation: (a) Body type recognition interface, (b) Grade difference generation interface, (c) Model change prediction interface, (d) Three-dimensional human body display interface.

\subsection{Female Body Shape Recognition Interface}

Mainly complete the classification and recognition of women's body types. In the main interface as shown in Figure 2a, first import the female net body size data (ORD file); then enter personal information such as female body identification number, name, age, hometown, height, weight, etc.; finally complete the query function and display Personal basic information, national standard number type, system classification number type, partial characteristics and body type evaluation, etc.

\subsection{Personalized Template Generation Interface}

It mainly completes the two functions of basic template generation and personalized template generation. In the basic model generation analysis, it includes the input interface of the upper garment size and the input interface of the lower garment size, which are used to store the basic pattern diagram of the garment manufacturing enterprise; the query interface of the pattern of the upper garment and the query interface of the pattern of the bottom garment. To query and form a basic model map. In the analysis of personalized template generation, it includes the ready-made garment grade difference generation interface (see Figure 2b) to generate the individual actual grade difference; the upper garment pattern change prediction interface and the bottom garment pattern change prediction interface (see Figure 2c). Eventually a personalized model of top and bottom garments is formed.

\subsection{Female Body Model Demonstration Interface}

The three-dimensional human body display interface (see Figure 2d) is used to view the female figure from all aspects and multiple angles. Through the left and bottom buttons, you can perform functions such as rotation, zoom, and surround on the female figure. On this basis, functions such as fitting on the human body can be further completed. 


\section{Conclusions}

In this paper, by collecting the body size of young women in Jiangsu and Zhejiang, a certain-scale women's net body size database is established, which can provide a reference for the establishment of a large-scale national body database. The SVM algorithm is used to identify and classify the main characteristic parameters of women's body shape, and finally it is determined to be 36 body types, and the analysis shows that the female body types in this area are mainly concentrated in 18 body types. The research results can be used as reference for clothing companies. Adopting J2EE architecture and selecting ECLIPSE development platform to establish a web-based female body shape recognition and model generation system, which has been trial-run in the enterprise, evaluated by experts from the project team, and the application effect is good; it can be also used for a wider range of network customers.

Acknowledgements. The paper is supported by Zhejiang Provincial Department of Education named Research and Design of 3D Human Body Fitting System Based on Immersive VR (Y201636734). The author would like to thank the 6 experts for their contributions to this project and the former researchers.

\section{References}

1. Dai Hong, Clothing size standard and its application. China Textile Publishing House, 1998, pp.38.

2. I S Yang, C K Chan, "Science Determines Sizes-establishment of a Sizing System for Clothing in North Korea", Textile Asia, vol. 11, no. 5, pp. 48-49, 1994.

3. Feng-yuan Zou,Su-yu Zhang,Xiao-jun Ding etc, "Female body shape recognition and application based on C/S mode", Journal of Textile Research, vol. 29, no. 6, pp. 101-104, 2008.

4. Feng-yuan Zou,Xiao-jun Ding,Li-feng Pan, "Research on Feature Index of Young Women's Body Shape and Ridge Regression Prediction", Journal of Textile Research, vol. 27, no. 4, pp. 56-59, 2006.

5. Su-yu Zhang, "Research on Young Females' Body Classification Based on SVM", International Journal of Science, vol. 7, no. 6, pp. 269-274, 2020. 